\section{Themenkomplex III: Therapie der nichterosiven Refluxkankheit (NERD)}

H. Koop, M. Classen, S. Liebe, A. Lütke, M. Wienbeck

Die nichterosive Refluxkrankheit (non-erosive reflux disease; NERD) ist mit einem geschätzten Anteil von ca. $60 \%$ die häufigste Manifestationsform der gastroösophagealen Refluxkrankheit. Ihr kommt somit eine große Bedeutung sowohl in medizinischer als auch ökonomischer Hinsicht zu. Ein Übergang von NERD zu einer Refluxösophagitis (erosive reflux disease; ERD) findet wahrscheinlich selten statt.

Im Gegensatz zur Häufigkeit der Erkrankung steht der Mangel an validen Studien zur Therapie von NERD: Anders als für die Refluxösophagitis sind für die NERD randomisierte Studien erst aus den letzten Jahren verfügbar, die ein verlässliches Urteil nur über Teilbereiche der Behandlung erlauben.

\section{Konsens}

In der Gesamtgruppe von Patienten mit Refluxsymptomen, aber unauffälliger Endoskopie verbergen sich unterschiedliche pathophysiologische Störungen bzw. Krankheitsentitäten (B).

\section{Kommentar}

Die NERD ist charakterisiert durch typische Refluxsymptome und dadurch bedingte eingeschränkte Lebensqualität, zugleich aber durch Fehlen entzündlicher Veränderungen in der ösophagealen Mukosa. Daher wird die Krankheitsentität allein durch das Beschwerdebild bestimmt. Da einzelne Refluxsymptome auch im Rahmen anderer Krankheitsbilder in unterschiedlicher Intensität und Häufigkeit auftreten, bestehen fließende Übergänge von NERD zum nichtkardialen Thoraxschmerz [1, 2], irritablen/hypersensitiven Ösophagus [3] und zu dem Reizmagen [4]. Die Ergebnisse einer säurehemmenden Therapie als etabliertem Prinzip bei der Refluxösophagitis fallen bei NERD sehr viel heterogener aus: Das Ansprechen auf eine Säurehemmung trotz geringerer Säureexposition des Ösophagus - ist weniger konsistent als bei einer Refluxösophagitis [5] und nach erfolgreicher Akuttherapie einer NERD (meist mit säurehemmenden Pharmaka) besteht im Weiteren auch unter Plazebo eine Rezidivfreiheit bei bis zu 50\% der Patienten über Zeiträume von 6-12 Monaten [6-8] im Gegensatz zu 10-15\% bei der Refluxösophagitis. Letzteres dürfte nicht allein einem intermittierenden Verlauf der NERD mit langen symptomfreien Intervallen zuzuschreiben sein, sondern vielmehr auch der Tatsache, dass in größerem Umfang Patienten in solche Studien eingeschlossen wurden, bei denen die Symptome nicht oder zumindest nur zu einem geringeren Teil durch Reflux induziert wurden [9]. Je subtiler ein pathologischer Reflux als Ursache der Beschwerdesymptomatik identifiziert werden kann (z.B. durch detaillierte Symptomerhebung bzw. ergänzende Methoden wie eine $\mathrm{pH}-$ Metrie), umso deutlicher wird der therapeutische Erfolg einer wirksamen säurehemmenden Therapie [3, 10]. Allerdings erscheint die mögliche Schlussfolgerung, zur weiteren Abgrenzung eine $\mathrm{pH}$-Metrie durchzuführen und somit Patienten mit pathologischem Reflux von solchen mit einem hypersensitiven Ösopha- gus zu unterscheiden, schon aus logistischen Gründen nicht praktikabel. Daher kommt der Probetherapie auch eine wichtige diagnostische Aussagekraft zu, ohne dass aus dem Ansprechen mit hinreichender Sicherheit auf die Diagnose einer NERD geschlossen werden kann (siehe Diagnostik).

\section{Konsens}

Die Lebensqualität ist bei NERD in gleicher Weise beeinträchtigt wie bei ERD (B).

\section{Kommentar}

Aufgrund der praktisch deckungsgleichen Symptome wird die Lebensqualität bei NERD in ähnlicher Weise beeinträchtigt [7, 11 - 14]: Die Intensität der einzelnen Symptome bei Refluxösophagitis durch NERD bestimmt die Beeinträchtigung der Lebensqualität, nicht aber Nachweis oder Fehlen peptischer Läsionen im Ösophagus [15]. Dies impliziert, dass durch die Therapie eine vollständige Wiederherstellung der Lebensqualität angestrebt werden sollte.

\section{Konsens}

Alleiniges Therapieziel ist die Beschwerdefreiheit des Patienten mit NERD (C).

\section{Kommentar}

Aufgrund des Fehlens von Läsionen steht die Beseitigung von Symptomen im Zentrum. Es ist nicht erforderlich, die Normalisierung der Säureexposition des Ösophagus bei asymptomatischen Patienten zu erfassen.

\section{Konsens}

Intoleranzen gegenüber bestimmten Nahrungs- und Genussmitteln sind zu berücksichtigen; die Empfehlung zu bestimmten Allgemeinmaßnahmen wie Gewichtsreduktion oder Erhöhung des Kopfendes des Bettes ist individuell zu erwägen. Längerfristiges Einhalten von Allgemeinmaßnahmen als Voraussetzung für die Gabe wirksamer medikamentöser Therapieverfahren ist abzulehnen $(\mathrm{C})$.

\section{Kommentar}

Derzeit sind keine Daten verfügbar, die die Wirksamkeit von Allgemeinmaßnahmen (Erhöhung des Kopfendes, diätetische Empfehlungen, Gewichtsreduktion etc.) allein bzw. insbesondere einen additiven Effekt solcher Empfehlungen zusätzlich zu einer medikamentösen Therapie bei NERD belegen (indem z. B. säurehemmende Pharmaka eingespart werden können). Die Wirksamkeit der einzelnen Maßnahmen allein ist gering und umstritten (Übersicht bei [16]). Dennoch können im Individualfall einzelne Maßnahmen nach ärztlicher Erfahrung hilfreich sein (Expertenmeinung). Viele Patienten haben aber solche Allgemeinmaßnahmen bereits vor dem Arztkontakt angewandt und diese als unwirksam bzw. begrenzt wirksam erfahren. Unter diesen Bedingungen ist eine alleinige Empfehlung zur Fortführung bzw. Intensivierung nicht sinnvoll und auch nicht Voraussetzung für den Beginn einer wirksamen medikamentösen Therapie.

\section{Konsens}

Sporadische Refluxbeschwerden, die gut auf Antazida ansprechen, können mittelfristig derart behandelt werden. Von mehr- 
mals täglichen Antazidagaben über einen längeren Zeitraum zur Symptomkontrolle wird dagegen abgeraten (C).

\section{Kommentar}

Daten zur Effektivität der Behandlung mit Antazida bei NERD fehlen: Zum einen haben die Patienten vor dem ersten Arztkontakt bereits selbst häufig zur Eigenmedikation mit Antazida gegriffen, zum anderen sind Antazida zumeist Reservemedikamente in Plazebo- wie auch Verumgruppen innerhalb von kontrollierten Studien [7, 8, 17]. Antazida sind - anders als in der Sicht vieler Patienten - keine inerten Substanzen, sondern vor allem bei längerem Einsatz und in höheren Dosen mit erheblichen Nebenwirkungen (unerwünschte Arzneimittelwirkungen) verbunden. Daher kann ihr Einsatz kann nur bei sporadischen Symptomen empfohlen werden. (Anmerkung: Treten Refluxsymptome nur sporadisch auf, ist dadurch die Lebensqualität meist nur marginal beeinflusst; damit läge definitionsgemäß keine Refluxkrankheit vor.) Mehrmals tägliche Einnahmen von Antazida deuten dagegen auf das Vorliegen einer Refluxösophagitis hin [18].

\section{Konsens}

Prokinetika stellen nach wie vor ein pathophysiologisch attraktives Therapieprinzip dar; mangels Verfügbarkeit effektiver Substanzen steht eine derartige Therapieform derzeit nicht zur Verfügung (A).

\section{Kommentar}

Über die Wirksamkeit von im Markt befindlichen Prokinetika (Metoclopramid, Domperidon) bei NERD sind keine aussagekräftigen Studien verfügbar, an ihrer therapeutischen Effektivität besteht großer Zweifel. Spezifische Therapieergebnisse zur Behandlung der NERD liegen nicht vor. Zu Cisaprid liegen validere Daten vor: Cisaprid ist hinsichtlich der Symptombeeinflussung $\mathrm{H}_{2}$-Blockern gleichwertig [20] und nicht signifikant besser als Plazebo [21], Protonenpumpenblockern aber deutlich unterlegen [21, 22]. Aufgrund von in Einzelfällen lebensbedrohlichen Arzneimittelinteraktionen ist diese Substanz derzeit nicht mehr verfügbar.

\section{Konsens}

Die Dosis-Wirkungs-Beziehung einer säurehemmenden Therapie ist - analog zu ERD - auch für NERD gültig (A).

\section{Kommentar}

Es liegen inzwischen eine Reihe von randomisierten Studien zur Effektivität der Behandlung von NERD mit verschiedenen säurehemmenden Pharmaka bzw. unterschiedlichen Dosen dieser Substanzen vor; teils wurden diese unterschiedlichen Substanzklassen gegeneinander vergleichend untersucht. Zusammengefasst ergibt sich folgendes Bild [23]: $\mathrm{H}_{2}$-Rezeptorantagonisten sind schwach wirksam [24], solide plazebokontrollierte Studien bei NERD sind aber kaum verfügbar. PPI sind deutlich wirksamer als Plazebo [3, 7, 15, 17, 21, 25 - 27], aber PPI sind gleichermaßen auch $\mathrm{H}_{2}$-Blockern überlegen $[5,11,14,26,28]$, wie dies auch in einer Metaanalyse gezeigt werden konnte [29]. Auch eine Erhöhung der $\mathrm{H}_{2}$-Blocker-Dosis führt zu keiner wesentlichen Steigerung der Effizienz [14, 30]. Ob innerhalb der Klasse der PPI höhere Dosen wirksamer sind als niedrige, ist bisher nicht abschließend beurteilbar $[5,8,10,15,25,26,30]$.

\section{Konsens}

Die Primärtherapie sollte mit einem PPI durchgeführt werden. Das Therapieresultat gibt zugleich auch diagnostische Informationen (A).

\section{Kommentar}

Aufgrund der überlegenen Wirkung der Säurehemmung mittels PPI gegenüber anderen Therapieformen ist zunächst eine probatorische Therapie mit einem Vertreter dieser Substanzklasse sinnvoll. Dies führt zu schnelleren und konsistenteren Therapieergebnissen [31]. Initial sollten Standarddosen verabreicht werden. Neben der überlegenen Wirksamkeit ergeben sich aus dem Therapieergebnis auch wichtige diagnostische Rückschlüsse (siehe dazu auch Probetherapie im Beitrag Diagnostik): Die PPITherapie ist insbesondere bei Patienten mit NERD und pathologischer $\mathrm{pH}$-Metrie wirksamer als bei solchen Patienten mit normaler pH-Metrie [10]. Über die optimale Dauer gibt es bisher keine einheitliche, durch Daten belegbare Auffassung, jedoch dürfte für die Mehrzahl der Patienten ein Behandlungsintervall von 4 Wochen zunächst ausreichen. Aufgrund der großen Variabilität der Rezidivrate bzw. des Fehlens von Prädiktoren für das drohende Rezidiv sollte nach Erreichen einer stabilen Beschwerdefreiheit die Therapie zunächst beendet werden.

\section{Konsens}

In der Mehrzahl der Fälle ist ein längerfristiges Therapiekonzept erforderlich. Dabei kann die Langzeitbehandlung in der Regel als „on demand“-Therapie mit Säuresekretionshemmern durchgeführt werden. Die Wirksamkeit eines solchen Therapiekonzeptes ist insbesondere für PPIs gut belegt (A).

\section{Kommentar}

Ähnlich der Refluxösophagitis (ERD) zeigt auch die NERD nach Absetzen eine hohe Rezidivrate. Daher ist in vielen Fällen die Implementierung einer Langzeitbehandlung notwendig.

In mehreren Studien ist die Wirksamkeit einer Behandlung mit PPIs on demand gegenüber Plazebo gesichert [6 - 8, 12, 27]. Ähnliche Untersuchungen für $\mathrm{H}_{2}$-Blocker existieren nicht, sondern nur in Form einer Dauertherapie [17, 30]. $\mathrm{H}_{2}$-Blocker sind PPIs unterlegen, wobei direkte Vergleiche für PPI gegen $\mathrm{H}_{2}$-Blocker in der Langzeittherapie unzureichend vorliegen [32, 33] und im Gegensatz zu PPI - täglich gegeben werden müssen. Soweit überhaupt quantitative Aussagen zum therapeutischen Gewinn über Plazebo hinaus möglich sind, so sind sie unter $10 \%$ anzunehmen. Wie lange eine Langzeittherapie bei NERD durchzuführen ist, ist durch Daten unzureichend belegt und von den Symptomen abhängig.

In der Praxis empfiehlt sich folgendes Vorgehen: Nach einer Phase der Akuttherapie mit einem PPI sollte die Behandlung zunächst ausgesetzt werden; der Patient wird aufgefordert, sich bei Wiederauftreten der Beschwerden erneut vorzustellen, um ihn dann bedarfsadaptiert mit einem PPI zu behandeln. Nach kontrollierten Studien ist im Mittel eine PPI-Dosis alle 2-3 Tage zur Erhaltung der Symptomfreiheit erforderlich [7, 8], während notwendige Dosierungsintervalle für $\mathrm{H}_{2}$-Blocker nicht bekannt sind. Wenn ein Patient gleichermaßen gut auf $\mathrm{H}_{2}$-Blocker wie auf PPI anspricht, kann die Therapie auch längerfristig mit einem $\mathrm{H}_{2}$-Blocker durchgeführt werden; ob eine solche $\mathrm{H}_{2}$-Blockerthe- 
rapie gegenüber einer „on demand“-Therapie mit einem PPI kosteneffizient ist, ist bisher nicht untersucht.

\section{Konsens}

Nur in Ausnahmefällen sollten Patienten mit NERD einer Antirefluxoperation zugeführt werden (C).

\section{Kommentar}

In kontrollierten Studien zur Beurteilung der Effektivität der Antirefluxchirurgie wurden nahezu ausschließlich Patienten eingeschlossen, bei denen zumindest im Vorfeld eine Refluxösophagitis dokumentiert worden war. Daten aus kontrollierten Studien zur Wirksamkeit operativer Verfahren bei NERD liegen dagegen nicht vor. Daher sollte große Zurückhaltung bei der Indikationsstellung zu einer Antirefluxoperation bei NERD gestellt werden. In jedem Falle wäre es unabdingbare Voraussetzung, dass präoperativ die pathophysiologische/n Störung/en identifiziert wird/werden, die auch durch einen operativen Eingriff korrigiert werden kann/können. Insbesondere das Versagen der konservativen Therapie als Indikation zur Operation ist abzulehnen, da sich - wie oben ausgeführt - die Differenzierung von Patienten mit NERD einerseits und funktionellen Beschwerden mit begleitendem Sodbrennen andererseits nicht immer sicher gelingt. Die Therapieergebnisse der operativen Therapie bei Patienten mit funktionellen Beschwerden dürften naturgemäß enttäuschend sein, während gute Symptombeeinflussung durch PPI präoperativ als ein Prädiktor für gute postoperative Ergebnisse gilt (siehe Beitrag Antirefluxchirurgie).

\section{Konsens}

Endoskopische Verfahren zur Verminderung von Refluxbeschwerden können derzeit außerhalb kontrollierter Studien nicht empfohlen werden, da insbesondere Vergleichsstudien zu etablierten medikamentösen oder chirurgischen Verfahren fehlen $(\mathrm{C})$.

\section{Kommentar}

In den letzten Jahren wurden unterschiedliche Methoden der endoskopischen Antirefluxtherapie entwickelt [34-37]: Neben Anlegen einer Naht im gastroösophagealen Übergang (entweder intramural oder transmural) existieren Untersuchungen zur intramuralen Injektion eines Polymers aus Acryl bzw. die Applikation thermischer Energie in der Kardia. Bislang ist unklar, welches Ziel mit der endoskopischen Therapie verfolgt wird: ob eine Reduktion des gastroösophagealen Refluxes, das künstliche Herbeiführen einer Stenose oder eine Denervierung der Kardia (mit konsekutiver Reduktion transienter Sphinkterrelaxationen und/oder herabgesetzter Schmerzperzeption) angestrebt würde [38]. Welche Mechanismen zur Symptombesserung welchen Methoden zugrunde liegen, ist bislang unklar. Auch sind die Risiken bisher nicht annähernd zu beschreiben (Todesfälle sind berichtet worden).

In einigen kontrollierten Studien ging kurzzeitig die Intensität der Symptome bzw. der quantitative Gebrauch säurehemmender Pharmaka in selektionierten Kollektiven zurück. Überwiegend wurden aber nur Patienten mit einer NERD oder geringen Schweregraden einer ERD eingeschlossen, die Größe der Hernie lag in der Regel unter $2 \mathrm{~cm}$. Daten zu Patienten mit mittleren und höheren Schweregraden fehlen. Zudem fehlen bislang vollkom- men vergleichende randomisierte Studien, in denen endoskopische Verfahren mit etablierten Behandlungskonzepten verglichen wurden (PPI-Therapie, Operation). Die bisher einzige randomisierte Studie - Applikation thermischer Energie in der Kardia („Stretta“) gegenüber der „Schein-Stretta“ - zeigte keine signifikanten Verbesserungen von pH-Metrie und Manometrie trotz nachgewiesener Beeinflussung der Refluxsymptomatik [39].

Bevor eine Stellungnahme zu den endoskopischen Techniken abgegeben werden kann, bedarf es intensiver kontrollierter Untersuchungen, in denen endoskopische Antirefluxmethoden mit einer fortgesetzten PPI-Behandlung und/oder einer operativen Antirefluxchirurgie verglichen werden. Solange solche Daten insbesondere zum Langzeitverlauf - nicht vorliegen, kann eine Anwendung endoskopischer Techniken außerhalb solcher Vergleichsstudien nicht empfohlen werden.

\section{Literatur}

${ }^{1}$ Fang J, Bjorkman D. A critical approach to noncardiac chest pain: pathophysiology, diagnosis and treatment. Am J Gastroenterol 2001; 96: $958-968$

${ }^{2}$ Achem SR, Kolts BE, MacMath T et al. Effects of omeprazole versus placebo in treatment of noncardiac chest pain and gastroesophageal reflux. Dig Dis Sc 1997; 42: 2138 - 2145

${ }^{3}$ Watson RGP, Tham TCK, Johnston BT et al. Double-blind cross-over placebo controlled study of omeprazole in the treatment of patients with reflux symptoms and physiological levels of acid reflux - the „sensitive oesophagus“. Gut 1997; 40: 587-590

${ }^{4}$ Carlsson R, Dent J, Bolling-Sternevald E et al. The usefulness of structured questionnaire in the assessment of symptomatic gastroesophageal reflux disease. Scand J Gastroenterol 1998; 33: 1023-1029

${ }^{5}$ Venables TL, Newland RD, Patel AC et al. Omeprazole 10 milligrams once daily, omeprazole $20 \mathrm{mg}$ once daily, or ranitidine 150 twice daily, evaluated as initial therapy for the relief of symptoms of gastrooesophageal reflux disease in general pratice. Scand J Gastroenterol 1997; 32: 965-973

${ }^{6}$ Venables TL, Newland RD, Patel AC et al. Maintenance treatment for gastro-oesophageal reflux. Scand J Gastroenterol 1997; 32: 627-632

${ }^{7}$ Lind T, Havelund T, Lundell L et al. On demand therapy with omeprazole for the long-term management of patients with heartburn without oesophagitis - a placebo-controlled randomised trial. Aliment Pharmacol Ther 1999; 13: 907-914

${ }^{8}$ Talley NJ, Lauritsen K, Tunturi-Hahnala $\mathrm{H}$ et al. Esomeprazole $20 \mathrm{mg}$ maintains symptom control in endoscopy-negative gastro-oesophageal reflux disease: a controlled trial of 'on-demand' therapy for 6 months. Aliment Pharmacol Ther 2001; 15: 347-354

${ }^{9}$ Martinez SD, Malagon IB, Garewal HS et al. Non-erosive reflux disease (NERD) - acid reflux and symptom patterns. Aliment Pharmacol Ther 2003; 17: $537-545$

${ }^{10}$ Lind T, Havelund T, Carlsson R et al. Heartburn without oesophagitis: efficacy of omeprazole therapy and features determining therapeutic response. Scand J Gastroenterol 1997; 32: 974 -979

${ }^{11}$ Lauritsen K. Management of endoscopy-negative reflux disease: progress with short-term treatment. Aliment Pharmacol Ther 1997; 11 (Suppl 2): 87-92

12 Lundell L. New information relevant to long-term management of endoscopy-negative reflux disease. Aliment Pharmacol 1997; 11 (Suppl 1): $93-98$

${ }^{13}$ Havelund T, Lind T, Wiklund I et al. Quality of life in patients with heartburn but without esophagitis: effects of treatment with omeprazole. Am J Gastroenterol 1999; 94: 1782 - 1789

${ }^{14}$ Wiklund I, Bardhan KD, Muller-Lissner S et al. Quality of life during acute and intermittent treatment of gastro-oesophageal reflux disease with omeprazole compared with ranitidine. Results from a multicentre clinical trial. The European Study Group. Ital J Gastroenterol 1998; 30: 19-27 
${ }^{15}$ Carlsson R, Dent J, Watts R et al. Gastro-oesophageal reflux disease in primary care: an international study of different treatment strategies with omeprazole. International GORD Study Group. Eur J Gastroenterol Hepatol 1998; 10: 119-124

${ }^{16}$ Meining A, Classen M. The role of diet and lifestyle measures in the pathogenesis and treatment of gastroesophageal reflux disease. Am J Gastroenterol 2000; 95: 2692-2697

17 Bate CM, Griffin SM, Keeling PWN et al. Reflux symptom relief with omeprazole in patients without unequivocal oesophagitis. Aliment Pharmacol Ther 1996; 10: 547 -555

${ }^{18}$ Graham DY, Smith JL, Patterson DJ. Why do apparently healthy people use antacid tablets? Am J Gastroenterol 1983; 78: 257-260

19 Simon B, Raveli GP, Goffin H. Sucralfate gel versus placebo in patients with non-erosive gastro-oesophageal reflux disease. Aliment Pharmacol Ther 1996; 10: $441-446$

${ }^{20}$ Reynolds JC. Individualized acute treatment strategies for gastrooesophageal reflux disease. Scand J Gastroenterol 1995; Suppl 213: $17-24$

${ }^{21}$ Hatlebakk JG, Hyggen A, Madsen PH et al. Heartburn treatment in primary care: randomised, double blind study for 8 weeks. Brit med J 1999; 319: 550-553

22 Galmiche JP, Barthelemy P, Hamelin B. Treating the symptoms of gastro-oesophageal reflux disease: a double-blind comparison of omeprazole and cisapride. Aliment Pharmacol Ther 1997; 11: 765-773

23 Pinxteren, Numans ME, Bonis PA et al. Short-term treatment with proton pump inhibitors, $\mathrm{H} 2$ receptor antagonists, and prokinetics for gastro-oesophageal reflux disease-like symptoms and endoscopy negative reflux disease. Cochrance Database Syst Rev 2000; 2: CD002095

${ }^{24}$ Sabesin SM, Berln RG, Humphries TJ et al. Famotidine relieves symptoms of gastroesophageal reflux disease and heals erosions and ulcerations. Results from a multicenter, placebo-controlled, dose-ranging study. Arch Intern Med 1991; 151: 2394-2400

${ }^{25}$ Richter JE, Kovacs TO, Greski-Rose PA et al. Lansoprazole in the treatment of heartburn in patient without erosive oesophagitis. Aliment Pharmacol Ther 1999; 13: 795-804

${ }^{26}$ Richter JE, Peura D, Benjamin SB et al. Efficacy of omeprazole for the treatment of symptomatic acid reflux disease without esophagitis. Arch Intern Med 2000; 160: 1810-1816
${ }^{27}$ Bytzer P, Blum A, de Herdt D et al. Six-month trial of on-demand rabeprazole $10 \mathrm{mg}$ maintains symptom relief in patients with non-erosive reflux disease. Aliment Pharmacol Ther 2004; 20: 181 - 188

${ }^{28}$ Armstrong D, Paré P, Pericak D et al. Symptom relief in gastroesophageal relux disease: a radomized, controlled comparison of pantoprazole and nizatidine in a mixed patient population with erosive esophagitis or endoscopy-negative reflux disease. Am J Gastroenterol 2001; 96: 2849-2857

${ }^{29}$ van Pinxteren B, Numans ME, Lau J et al. Short-term treatment of gastroesophageal reflux disease. J Gen Inern Med 2003; 18: 755 - 763

${ }^{30}$ Kharilas PJ, Fennerty MB, Joelsson B. High- versus standard-dose ranitidine for control of heartburn in poorly responsive acid reflux disease: a prospective, controlled trial. Am J Gastroenterol 1999; 94: 92 - 97

${ }^{31}$ Armstrong D, Talley NJ, Lauritsen $\mathrm{K}$ et al. The role of acid suppression in patients with endoscopy-negative reflux disease: the effect of treatment with esomeprazole or omeprazole. Aliment Pharmacol Ther 2004; 20: $413-421$

${ }^{32}$ Howden CW, Henning JM, Huang B et al. Management of heartburn in a large, randomized, community-based study: comparison of four therapeutic strategies. Am J Gastroenterol 2001; 96: 1704 - 1710

${ }^{33}$ Bardhan KD, Müller-Lissner S, Bigard MA et al. Symptomatic gastrooesophageal reflux disease: double blind controlled study of intermittent treatment with omeprazole and ranitidine. Brit med J 1999; 318 : $502-507$

${ }^{34}$ Filipi CJ, Lehman GA, Rothstein RI et al. Transoral flexible endoscopic sturing for treament of GERD: a multicenter trial. Gastrointest Endosc 2001; 53: 416-422

${ }^{35}$ Chuttani R, Sud R, Sachdev G et al. A novel endoscopic full-thickness plicator for the treatment of GERD: a pilot study. Gastrointest Endosc 2003; 58: 770-776

${ }^{36}$ Triadafilopouslos G, DiBaise JK, Nostrant TT et al. The Stretta procedure for the treatment of GERD: 6 and 12 month follow-up of the U.S. open label trial. Gastrointest Endosc 2002; 55: 149-156

37 Johnson DA, Ganz R, Aisenberg J et al. Endoscopic implantation of enteryx for treatment of GERD: 12-month results of a prospective, multicenter trial. Am J Gastroenterol 2003; 98: 1909-1912

38 Kahrilas PJ. Radiofrequency energy tretment of GERD. Gastroenterology 2003; 125: 970-973

39 Corley DA, Katz P, Wo JM et al. Improvement of gastroesophageal reflux symptoms after radiofrequency energy: a randomized, shamcontrolled trial. Gastroenterology 2003; 125: 668 - 676

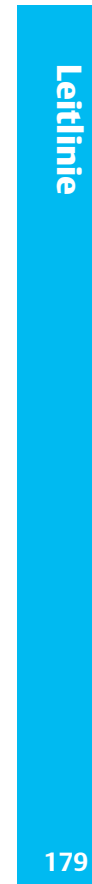

